\title{
Erratum to: Greater occipital nerve infiltration under MR guidance: Feasibility study and preliminary results
}

\author{
Adrian Kastler ${ }^{1,2} \cdot$ Romain Perolat $^{1,2} \cdot{\text { Bruno } \text { Kastler }^{3} \text { - Caroline Maindet-Dominici }}^{4}$. \\ Jan Fritz $^{5}$ - Alim Louis Benabid ${ }^{2}$ - Stephan Chabardes ${ }^{2,6}$ - Alexandre Krainik ${ }^{1}$
}

Published online: 13 October 2017

(C) European Society of Radiology 2017

\section{Erratum to: Eur Radiol \\ https://doi.org/10.1007/s00330-017-4952-3}

The original version of this article, published on 12 July 2017 , unfortunately contained mistakes. The following corrections have therefore been made in the original:

Figures $2-5$ were in a wrong position. They are reproduced here with the correct captions.

Fig. 2 Diagram illustrating MRguided greater occipital nerve (GON) infiltration procedural steps

\begin{tabular}{|c|c|}
\hline \multirow[b]{2}{*}{ Planning Phase } & Localizer \\
\hline & Sequence 1 (Ax. PD C1-C2) $\longrightarrow$ Target Planning on Obtained Images \\
\hline \multirow[t]{3}{*}{ Body Coil } & Coil change \\
\hline & Sequence 2 (Ax. PD) $\longrightarrow$ Choice of Gadolinium marker for needle entry point selection \\
\hline & Needle Insertion \\
\hline Interventional Phase & $\longrightarrow$ Needle progression control. This sequence may be repeated \\
\hline \multirow{4}{*}{ Loop Coil } & Saline injection \\
\hline & Sequence 3 (Ax. PD SPAIR) $\longrightarrow$ Control of accurate diffusion \\
\hline & LA and Steroid injection \\
\hline & Coil change \\
\hline Control Phase & Sequence 3 (Ax/Sag. PD SPAIR) $\rightarrow$ Control of accurate diffusion of injectant \\
\hline Body Coil & \\
\hline
\end{tabular}

The online version of the original article can be found at https:// doi.org/10.1007/s00330-017-4952-3

Adrian Kastler

Kastler.radio@gmail.com

1 Neuroradiology and MRI Unit, Grenoble Alpes University Hospital, CS 10217, F-38043 Grenoble Cedex 9, France

2 CLINATEC Research Facility, LETI, CEA Grenoble, Grenoble, France

3 Adult Radiology Department, Necker Hospital, Paris V University, Paris, France
4 Pain Management Unit, Grenoble Alpes University Hospital, Grenoble, France

5 Russell H. Morgan Department of Radiology and Radiological Science, The Johns Hopkins University School of Medicine, Baltimore, MD, USA

6 Neurosurgery Unit, Grenoble Alpes University Hospital, Grenoble, France 

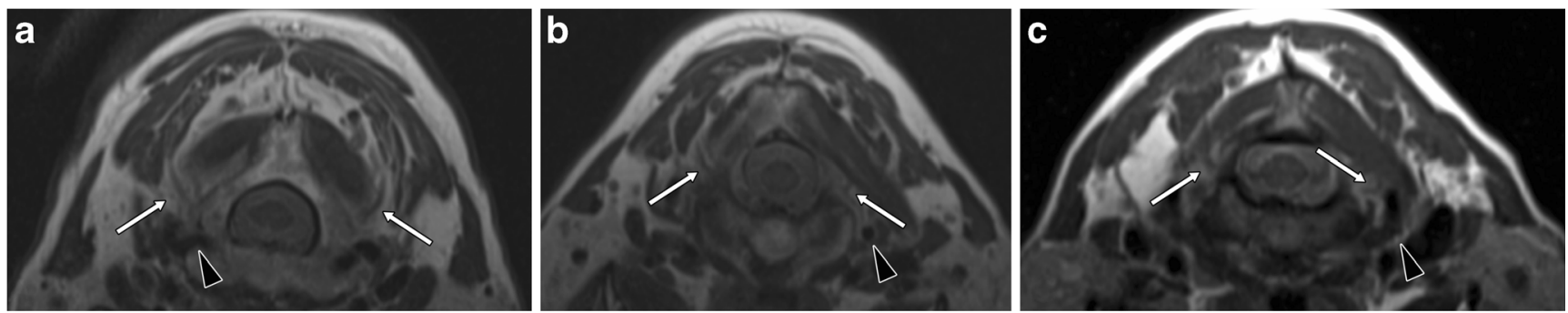

Fig. 3 MR images depicting the visualisation of the greater occipital nerve (GON). (a, b and c) These images are extracted from different patients' scanners, but all depict the exit of the GON nerve (white arrow) after the C1-C2 foramen, and its close relationship with the vertebral artery

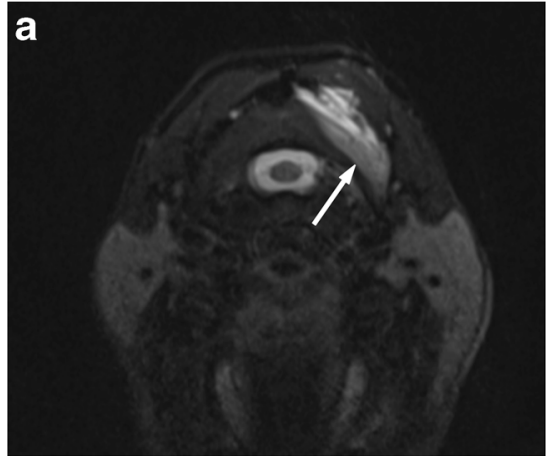

Fig. 4 Example of intra-inferior obliquus muscle diffusion. Axial and sagittal proton density (PD)-weighted Spectral Attenuated Inversion Recovery (SPAIR) images after greater occipital nerve (GON) infiltration showing diffusion in the obliquus inferior muscle (white

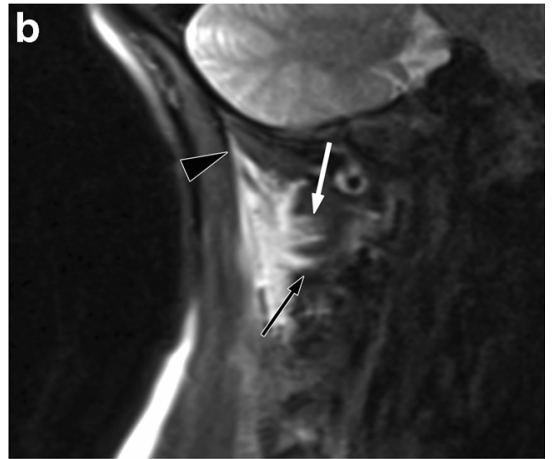

arrow). Sagittal image shows a perfect diffusion of the products from the exit of the nerve after the foramen under the obliquus inferior muscle (black arrow) to the semispinalis penetration area of the GON (white arrowhead)

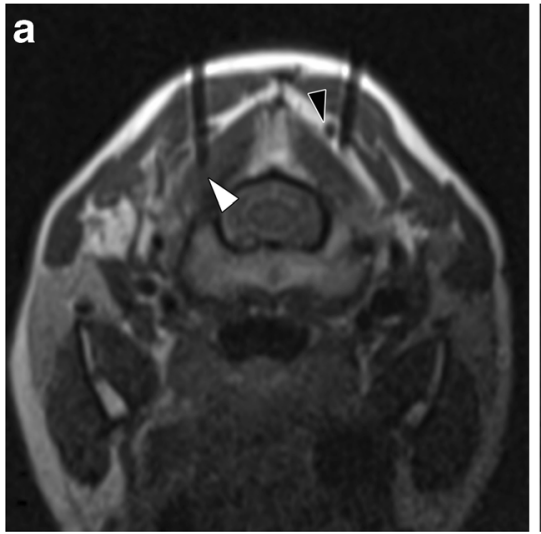

Fig. 5 Example of intraprocedural images showing the accuracy of MR guidance. (a) The accurate visualisation of the needle tip situated in close proximity to an avoided vascular structure (black arrow) and a misplacement of the right needle tip situated intra-muscularly to the

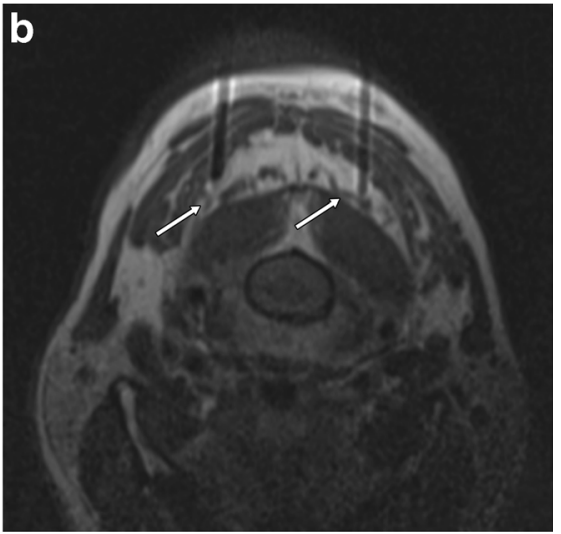

obliquus inferior (white arrowhead). (b) The visualisation of the greater occipital nerve (GON) during the needle placement, allowing for the needle tip to be placed immediately next to the GON nerves (white arrows) 